Article

\title{
Decursinol Angelate Inhibits LPS-Induced Macrophage Polarization through Modulation of the NFKB and MAPK Signaling Pathways
}

\author{
Salman Ul Islam ${ }^{1,+}$, Jung Ho Lee ${ }^{1, \dagger}{ }^{,}$Adeeb Shehzad ${ }^{2}$, Eun-Mi Ahn ${ }^{3}$, You Mie Lee ${ }^{4}$ and \\ Young Sup Lee ${ }^{1, *}$ \\ 1 School of Life Sciences, BK21 Plus KNU Creative BioResearch Group, Kyungpook National University, \\ Daegu 41566, Korea; salman2013@knu.ac.kr (S.U.I.); quiet93@dgist.ac.kr (J.H.L.) \\ 2 Department of Biomedical Engineering and Sciences, School of Mechanical and Manufacturing Engineering, \\ National University of Sciences and Technology, Islamabad 44000, Pakistan; \\ adeeb.shehzad@smme.nust.edu.pk \\ 3 Division of Biotechnology and Convergence, Daegu Haany University, Daegu 38610, Korea; \\ ahnEM@dhu.ac.kr \\ 4 College of Pharmacy, National Basic Research Laboratory of Vascular Homeostasis Regulation, \\ Kyungpook National University, Daegu 41566, Korea; lym@knu.ac.kr \\ * Correspondence: yselee@knu.ac.kr; Tel.: +82-53-950-6353; Fax: +82-53-943-2762 \\ + These authors contributed equally to this work.
}

Received: 4 July 2018; Accepted: 26 July 2018; Published: 27 July 2018

\begin{abstract}
Inflammation is considered the root cause of various inflammatory diseases, including cancers. Decursinol angelate (DA), a pyranocoumarin compound obtained from the roots of Angelica gigas, has been reported to exhibit potent anti-inflammatory effects. In this study, the anti-inflammatory effects of DA on the MAP kinase and NFKB signaling pathways and the expression of pro-inflammatory cytokines were investigated in phorbol 12-myristate 13-acetate (PMA)-activated human promyelocytic leukemia (HL-60) and lipopolysaccharide (LPS)-stimulated macrophage (Raw 264.7) cell lines. PMA induced the activation of the MAP kinase-NFkB pathway and the production of pro-inflammatory cytokines in differentiated monocytes. Treatment with DA inhibited the activation of MAP kinases and the translocation of $\mathrm{NF} k \mathrm{~B}$, and decreased the expression and exogenous secretion of IL-1 $\beta$ and IL-6. Furthermore, LPS-stimulated Raw 264.7 cells were found to have increased expression of M1 macrophage-associated markers, such as NADPH oxidase (NOX) and inducible nitric oxide synthase (iNOS), and the M2 macrophage-associated marker CD11b. LPS also activated pro-inflammatory cytokines and Erk-NFkB. Treatment with DA suppressed LPS-induced macrophage polarization and the inflammatory response by blocking Raf-ERK and the translocation of NFKB in Raw 264.7 cells. Treatment with DA also inhibited the expression of pro-inflammatory cytokines, such as IL-1 $\beta$ and IL-6, NOX, and iNOS in Raw 264.7 cells. These results suggest that DA has the potential to inhibit macrophage polarization and inflammation by blocking the activation of pro-inflammatory signals. These anti-inflammatory effects of DA may contribute to its potential use as a therapeutic strategy against various inflammation-induced cancers.
\end{abstract}

Keywords: inflammation; cytokines; MAP kinase; NFkB; decursinol angelate

\section{Introduction}

Inflammation, the immediate response of the body to a damage, is triggered by noxious stimuli and conditions, such as infection and tissue injury [1]. Inflammation is a complex physiological process that can promote cancer development through abnormal gene regulation and signal transduction. 
There is growing evidence that many cancers are initiated by infections, and it has been estimated that among 1.2 million cases of cancer annually, 15\% can be due to infections [2-5]. Within the host, persistent infections induce chronic inflammation, which subsequently, through multiple steps like generation of reactive oxygen and nitrogen species, DNA damage, and permanent genomic alterations (point mutations, deletions, or rearrangements), leads to cancer [6,7]. It is widely accepted that there is a causal relationship between inflammation, innate immunity and cancer; however, many underlying molecular and cellular mechanisms are still not clear. Tumor-associated macrophages (TAMs), which are found in the tumor microenvironment, are extremely differentiated cells derived from circulating monocytes. They exhibit pro-cancerous effects that are often related to tumor growth and invasion in cancer patients with poor clinical outcomes [8]. TAM activation or macrophage polarization by inflammatory cytokines (IFN- $\gamma$, TNF, and IL-12), anti-inflammatory cytokines (IL-4 and IL-10), or bacterial lipopolysaccharide (LPS) is the primary process involved in inflammation-associated cancers. This process results in the loss of homeostatic tissue architecture followed by immune suppression $[9,10]$. There is compelling evidence that immune responses are mediated by two main phenotypes of macrophages; M1 (classical) and M2 (alternative) [11,12]. M1 macrophages are activated by LPS, IFN- $\gamma$, and TNF- $\alpha$, and are associated with the expression of cytokines such as IL-1 $\beta$, IL- 6 , IL-9, IL-13, and inducible nitric oxide synthase (iNOS) in cancer patients $[13,14]$. The alternative M2 macrophages are associated with tumor progression and cell survival in cancer patients. Conversely, M2 macrophages have been reported to inhibit inflammation by producing anti-inflammatory cytokines, such as IL-4 and IL-10, that reduce the expression of iNOS and T cell proliferation [13,14]. M2 macrophages also mediate the disruption of the extracellular matrix (ECM) through increased vascularization [15].

Leukemia is a cancer of blood-forming cells usually present in the bone marrow. According to the American Cancer Society, a total of 62,130 new cases and 24,500 deaths caused by leukemia were expected in 2017 [16]. The role of inflammation has been demonstrated in leukemia. Toll-like receptor 4 (TLR4) is the main recognition receptor expressed in human monocytes and is abnormally regulated during inflammation [17]. Additionally, TLR4 has been found to be over-expressed in ulcerative colitis patients [18]. Upon activation, TLR4 augments macrophage activity through inflammatory cytokines, including TNF- $\alpha$, IL-6, and IL- $1 \beta$, the nuclear translocation of nuclear factor $\kappa B(N F \kappa B)$, and the activation of mitogen-activated protein kinases (MAPKs) [19]. Studies have also reported that MAPK activation is involved in LPS-induced nitric oxide synthase (iNOS) expression, at least in part through the modulation of p38 and JNK in RAW 264.7 macrophages [20]. Activation of iNOS has also been shown to enhance inflammatory bowel disease by releasing excessive nitric oxide (NO), which erodes colon integrity via the synthesis of peroxynitrite, a potent oxidizing agent formed during the reaction of NO with the superoxide anion [21]. Inflammatory diseases are complicated, and detailed studies are needed to discover new treatment modalities.

Decursinol angelate (DA) is a pyranocoumarin compound isolated from the roots of the Korean medicinal herb Angelica gigas Nakai, which belongs to the family Umbelliferae. Traditionally, this herb has been used as an immune-booster in the treatment of gynecological disorders and anemia [22,23]. Several studies have characterized the anti-inflammatory, anticancer, and anti-angiogenic properties of decursin in various cancers, including myelogenous leukemia cell lines [24]. Shehzad et al. reported that decursin and DA are the potent candidates for the treatment of various chronic inflammatory diseases such as diabetic retinopathy, osteoclastogenesis, rheumatoid arthritis, cancers (leukemia, myeloma, breast, prostate, and cervical), allergy, hepatic fibrosis, and Alzheimer's disease [25]. Studies have also reported that decursin inhibits matrix metallopeptidase 9 (MMP-9)-induced cytoskeletal rearrangement by suppressing phosphoinositide 3-kinase (PI3K), extracellular signal-regulated kinase (ERK), and NFKB activation in fibrosarcoma and breast cancer cell lines [26]. Decursin has also been shown to reduce the phosphorylation of ERK and JNK, but not p38 MAPK, in vascular endothelial growth factor (VEGF)-stimulated HUVECs [27]. 
Despite the well-documented anti-inflammatory effects of DA, the mechanisms underlying these effects are not yet fully understood. Therefore, the current study was conducted to elucidate the mechanism underlying the anti-inflammatory effects of DA on macrophage polarization. DA inhibited the differentiation and polarization of macrophages by suppressing the expression of inflammatory cytokines and the MAPK and NFKB pathways, as well as by reducing NADPH oxidase (NOX) and iNOS levels in LPS and TPA-induced inflammatory models.

\section{Results}

\subsection{DA Inhibits Cytokine Production in PMA-Induced Macrophages}

Macrophages produce many pro-inflammatory cytokines; among those produced by M1 macrophages are IL-1, IL-4, IL-6, IL-12, and TNF $\alpha$, while those produced by M2 macrophages include IL-10 and very low level of IL-12 [28]. Macrophages play a differential role in tumor growth and malignancy $[28,29]$. To evaluate the effects of DA treatment on cytokine production in PMA-stimulated macrophages, we analyzed the mRNA levels of IL-1 $\beta$, IL-4, IL-6, and IL-10 through RT-PCR using RNA from DA-treated HL-60 cell lines as the template. PMA upregulated the mRNA levels of IL-1 $\beta$ and IL-6, whereas treatment with DA reduced the levels of IL-1 $\beta$ and IL-6 mRNA (Figure 1A). However, neither PMA nor DA affected the expression of IL-4 and IL-10 at the mRNA level (Figure 1A). We further evaluated the exogenous secretion of IL-1 $\beta$ after exposure to PMA and DA. As shown in Figure 1B, treatment with DA effectively inhibited the PMA-induced activation of IL-1 $\beta$. Furthermore, to determine whether there is a synergistic effect between IL- $1 \beta$ and PMA that induces maximal IL-6 secretion, we exposed HL-60 cells to $5 \mathrm{ng} / \mathrm{mL}$ of IL-1. As shown in Figure 1C, the mRNA expression of IL-6 increased upon exposure to IL-1 $\beta$ and PMA alone, as well as their concomitant treatment, and decreased upon treatment with DA. Previous studies have shown that external stimuli, such as elevated temperature, induce the expression of IL-6. As shown in Figure 1D, IL-1 $\beta$ and PMA increased the expression and secretion of IL-6 into the culture media as measured through ELISA. DA treatment significantly reduced the IL- $1 \beta$-induced secretion of IL- 6 by PMA-induced differentiated cells. These data suggest that DA has the potential to inhibit the expression of pro-inflammatory cytokines in PMA-induced macrophages.

\subsection{DA Inhibits PMA-Induced NFKB and MAPK Activation in Macrophages}

The inflammatory cytokine IL-1 is responsible for the induction of IL-6 expression and the activation of MAPKs and NFKB in various inflammatory diseases [30]. NFKB is a transcription factor with pro-tumorigenic activity and is capable of evoking an inflammatory response. To study the anti-inflammatory mechanism of DA in macrophage polarization, HL-60 and Raw 264.7 cells were incubated with $100 \mathrm{ng} / \mathrm{mL}$ of PMA for $12 \mathrm{~h}$ and then treated with varying concentrations of DA



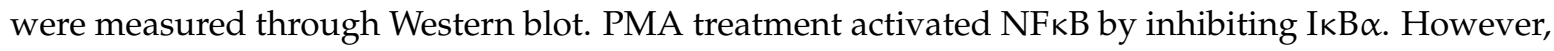
DA treatment significantly suppressed the activation of the NFKB p65 subunit in both HL-60 and Raw 264.7 cells (Figure 2A). Quantitative analysis of the Western blot data also revealed that PMA increased the expression of NFkB p65 in both HL-60 and Raw 264.7 cell lines (Figure 2B). DA suppressed the PMA-induced activation of $\mathrm{NF} \kappa \mathrm{B}$ by stabilizing $\mathrm{I} \kappa \mathrm{B} \alpha$ in macrophages. Proteins in the MAPK family are also involved in inflammatory diseases through their activation of inflammatory mediators. Drugs and other agents that inhibit this pathway are likely to have anti-inflammatory effects and may be of therapeutic interest. To determine the role of the MAPK pathway in PMA-induced macrophage polarization, we measured the expression levels of phosphorylated Raf, MEK, and ERK in cells that have been exposed to PMA and DA. PMA treatment increased the phosphorylation of Raf, MEK, and ERK in both HL-60 and Raw 264.7 cell lines (Figure 2C). However, DA treatment reduced phosphorylation and reversed the PMA-mediated activation of the MAPK pathway (Figure 2C). DA treatment did not significantly affect the p38/JNK pathway in HL-60 and Raw 264.7 cell lines 
(Figure 2D). These results indicate that DA may block the PMA-induced differentiation of macrophages by controlling the expression level of MAPK and NFKB.



Figure 1. DA inhibits PMA-induced production of proinflammatory cytokines. (A) total RNA was extracted from the treated HL-60 cells using TRIZOL reagent and was reverse-transcribed into cDNA. The amplified products were analyzed through electrophoresis on a $1 \%$ agarose gel and visualized using an EcoDye ${ }^{\mathrm{TM}}$ Nucleic Acid Staining Solution. Images were then captured using Wise Capture I-1000 software (Daihan Scientific, Seoul, South Korea). DA inhibited pro-inflammatory cytokines in PMA-induced macrophages; (B) IL-1 $\beta$ exogenous secretion was measured through ELISA. A 96-well plate was coated with $10 \mu \mathrm{g} / \mathrm{mL}$ purified goat anti-IL-1 $\beta$ antibody. Cell culture supernatants from the control and treated groups were incubated in these plates for $1 \mathrm{~h}$, and then a polyclonal rabbit anti-IL-1 $\beta$ antibody was added to the wells. HRP-conjugated goat anti-rabbit IgG was then added, and the cells were incubated for $30 \mathrm{~min}$. ABTS solution was then added to the plate, and the signal that was developed was detected using a reference wavelength of $490 \mathrm{~nm}$. Data are given as the mean \pm SD. ${ }^{*} p<0.05,{ }^{* *} p<0.01,{ }^{* * *} p<0.001$; (C) cells were treated with PMA overnight and then incubated with IL-1 $\beta$, PMA, or $30 \mu \mathrm{M}$ DA for the specified amount of time. RT-PCR was performed to analyze the expression of IL- 6 at the mRNA level. PMA and IL-1 $\beta$ upregulated the mRNA expression of IL-6, which was effectively reduced by DA. GAPDH was used as the reference gene; (D) the exogenous secretion of IL- 6 was analyzed through ELISA as described for IL- $1 \beta$. PMA and IL- $1 \beta$ were found to exert a synergistic effect, while treatment with DA inhibited the exogenous secretion of IL-6. Data are given as the mean $\pm \mathrm{SD}$. ${ }^{* * *} p<0.001$. 
(
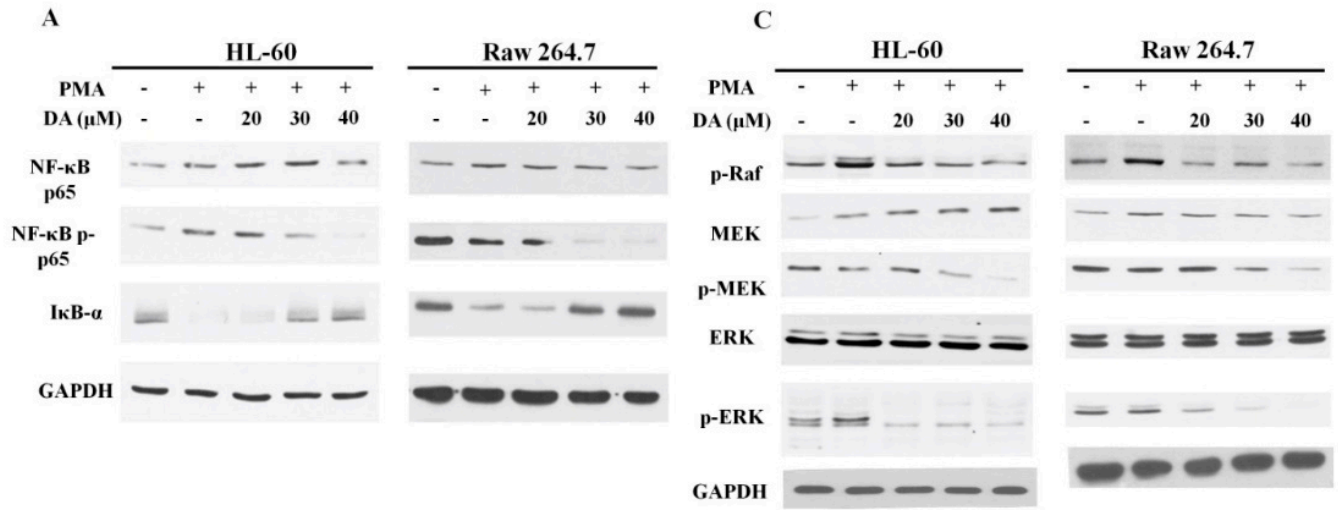

B
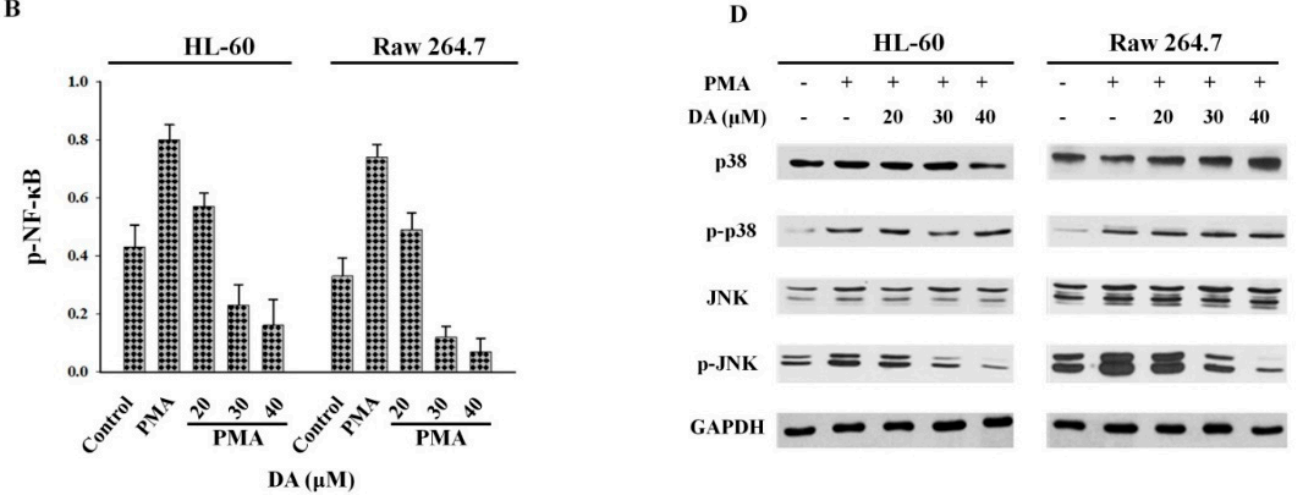

Figure 2. DA inhibits the NFKB and MAPK signaling pathways in PMA-induced macrophages. (A) treatment with DA abolished the activation of the NFkB pathway in PMA-induced macrophages. Cells were treated with PMA for $12 \mathrm{~h}$ in the dark and then incubated with different concentrations of DA $(0,20,30,40 \mu \mathrm{M})$ for the indicated amounts of time. Cell lysates were separated through $10 \%$ SDS-PAGE, and proteins were detected using an ECL Western blotting detection reagent. GAPDH was used as internal control; (B) NFkB phosphorylation was quantified using the software Image J. The results from three independent experiments are presented in a bar chart; (C) DA inhibits the activation of the ERK and JNK pathways. Cells were incubated with PMA or DA for the specified amounts of time, and the levels of total and phosphorylated proteins involved in the MAPK pathway were analyzed through Western blot. GAPDH was used as internal control; (D) cells were incubated with either PMA or DA for specified interval of time and MAPK pathway total and/or phosphorylated proteins were analyzed by Western blot.

\subsection{Effect of DA on LPS-Induced M1 Macrophages}

LPS has been reported to induce M1 macrophages, which results in higher levels of pro-inflammatory cytokines that dictate inflammatory $\mathrm{T}$ cell responses. Moreover, stimulation by LPS can transform M2 macrophages into M1 macrophages, thereby promoting inflammation [31]. To further elucidate the mechanisms underlying the activity of DA against LPS-induced macrophage polarization, Raw 264.7 cells were treated with bacterial LPS and the expression levels of inflammatory cytokines, such as IL-1 $\beta$ and IL-6, were measured. Treatment with LPS increased the expression of both IL-1 $\beta$ and IL- 6 at the mRNA level, which was effectively reversed by treatment with $30 \mu \mathrm{M}$ DA (Figure $3 \mathrm{~A}$ ). DA treatment also reduced the secretion of IL-1 $\beta$ and IL-6 by LPS-stimulated Raw 264.7 cells (Figure 3B). Since NOX-mediated iNOS production plays an important role in differentiation and macrophage polarization, we examined the mRNA expression of the M1 macrophage markers NOX and iNOS in LPS-stimulated Raw 264.7 cells through RT-PCR. As shown in Figure 3C, NOX and iNOS expression increased noticeably in cells treated with LPS $(100 \mathrm{ng} / \mathrm{mL})$. However, DA treatment abolished the LPS-induced NOX and iNOS expression (Figure 3C). In line with these results, we also performed immunofluorescence experiments to investigate the effect of DA on macrophage polarization. 
In these experiments, we used iNOS and CD11b as markers for the M1 and M2 phenotypes, respectively (Figure 3D). Microscopic analysis revealed that exposure to LPS increased the number of CD11b- and iNOS-positive cells as compared to the control set-up. DA treatment reduced the number of CD11b-and iNOS-positive cells and, consequently, inhibited macrophage polarization (Figure 3D). These results are consistent with those of the RT-PCR analysis, where DA was found to inhibit iNOS mRNA expression.

A

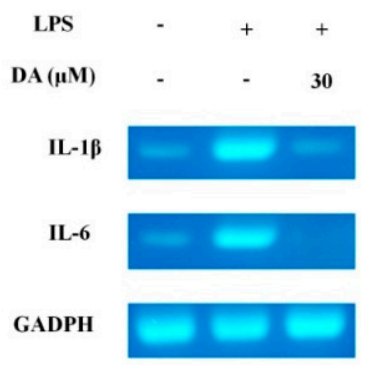

C

iNOS

GADPH

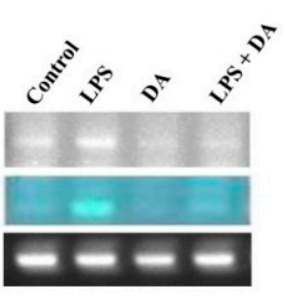

B

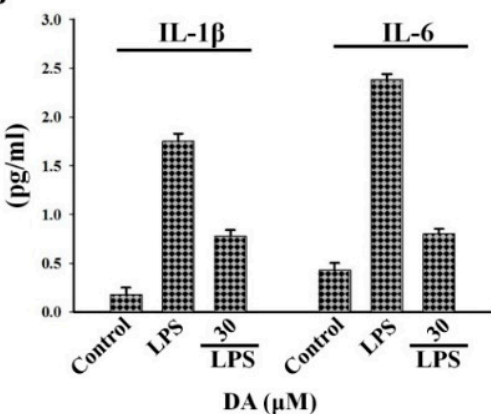

D

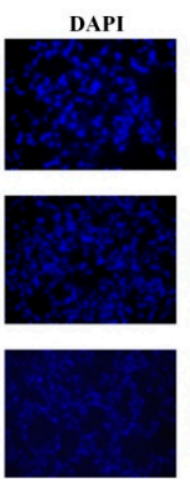
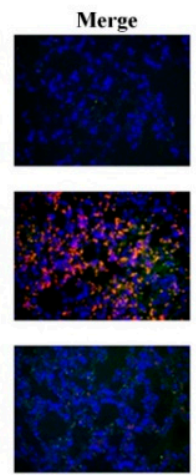

Figure 3. DA inhibits macrophage polarization. (A) mRNA levels of IL- $1 \beta$ and IL-6 were analyzed through RT-PCR after incubating the cells with LPS and $30 \mu \mathrm{M}$ DA; (B) the exogenous secretion of IL-1 $\beta$ and IL- 6 by LPS-stimulated cells was analyzed through ELISA. LPS stimulated the secretion of IL-1 $\beta$ and IL-6, which was significantly blocked by DA; (C) mRNA levels of NOX and iNOS in cells incubated with LPS with or without DA were analyzed through RT-PCR. (D) RAW 264.7 cells were seeded onto a 4 -well chamber slide and cultured until $70 \%$ confluency was reached. The cells were then incubated with LPS with or without DA for the indicated amounts of time. Cells were fixed with $3.7 \%$ formaldehyde and then permeabilized by incubating them with $0.1 \%$ Triton X-100 for $4 \mathrm{~min}$. Afterwards, the cells were incubated with anti-CD11b or anti-iNOS antibodies overnight and then incubated with FITC- or Alexa Flour 594-conjugated antibodies. Finally, the cells were incubated with DAPI mounting medium for 5-10 min and analyzed through confocal microscopy.

\subsection{DA Suppresses LPS-Induced MAPK and NFאB Activation in Raw 264.7 Cells}

There is compelling evidence that the activation of the MAPK and NFkb pathways is crucial for the induction of the expression of pro-inflammatory cytokines upon exposure to LPS. To determine whether LPS-induced macrophage polarization is mediated by the activation of NFKB (p50 and p65), Raw 264.7 cells were exposed to LPS and then treated with DA. To determine whether NFkB was translocated from the cytoplasm to the nucleus, we prepared cytosolic and nuclear fractions of Raw 264.7 cells. As shown in Figure 4A, LPS treatment induced the activation of NFkB, particularly of the subunits p50 and p65, which was effectively blocked by treatment with DA. Fluorescence microscopy is often used to identify drug targets by examining the sub-cellular localization of target proteins in inflammatory disorders. Therefore, we performed fluorescence microscopy to evaluate 
the translocation of NFkB from the cytoplasm to the nucleus. As shown in Figure 4B, LPS treatment resulted in the translocation of $\mathrm{NF} \times \mathrm{B}$ from the cytoplasm to the nucleus as indicated by the detection of the FITC-conjugated anti-NFkB antibody and the blue fluorescence of nuclear counterstain DAPI. In contrast, the translocation of $\mathrm{NF} \times \mathrm{B}$ was reduced in DA-treated cells (Figure $4 \mathrm{~B}$ ). The activation of MAPKs has been shown to play a central role in LPS-induced cytokine production during inflammation. Through the results we obtained, we have demonstrated the inhibitory effect of DA on the LPS-induced activation of the MAPK pathway (Figure 4C).
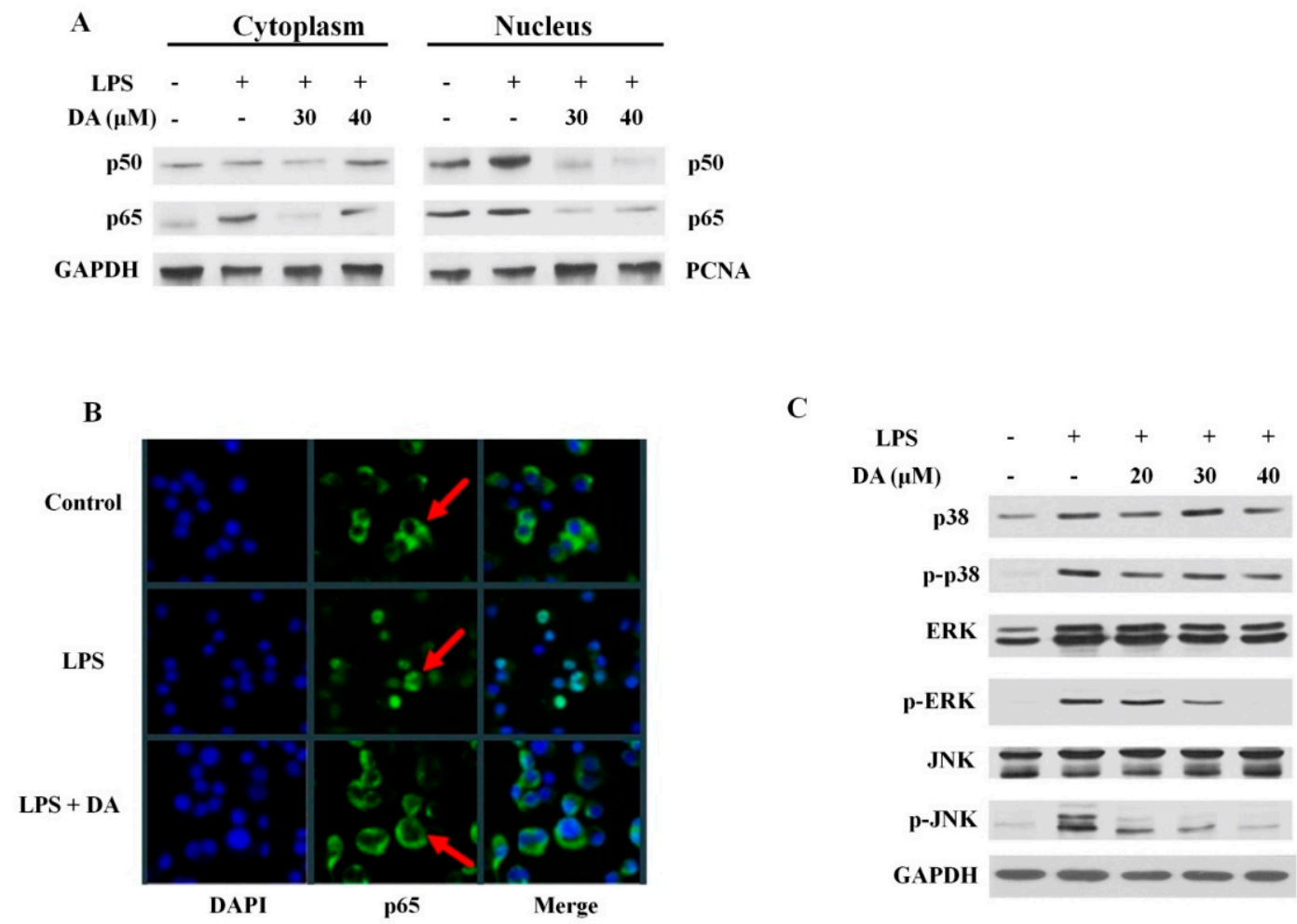

Figure 4. DA inhibits the activation of the NFKB and MAPK pathways by LPS in Raw 264.7 cells. (A) cells were stimulated with LPS and then incubated with or without DA for the indicated amounts of time. Cytoplasmic and nuclear fractions and total cell lysates were analyzed through a Western blot assay. DA inhibited the activation of the NFKB and MAPK pathways by LPS in Raw 264.7 cells; (B) nuclear localization of the p65 subunit of $\mathrm{NF}_{\mathrm{K} B}$ was analyzed through immunofluorescence. Briefly, cells were fixed with $3.7 \%$ formaldehyde and permeabilized by incubating them with $0.1 \%$ Triton $\mathrm{X}-100$. The cells were incubated with anti-NFKB p65 antibodies overnight at $4{ }^{\circ} \mathrm{C}$, and then with goat anti-rabbit IgG-FITC for $1 \mathrm{~h}$ at room temperature in the dark. Next, the cells were incubated with DAPI mounting medium for $10 \mathrm{~min}$ and analyzed using a confocal microscope. Fluorescent images of the cytoplasmic and nuclear fractions were merged to locate p65. DA inhibited the translocation of p65 into the nucleus; (C) DA inhibits the activation of the MAPK pathway. Cells were incubated with LPS with or without DA for the specified amounts of time, and the levels of total and/or phosphorylated proteins involved in the MAPK pathway were analyzed through Western blot.

\section{Discussion}

It has long been established that inflammation and infections are strongly correlated with the development of various cancers and malignancies [2-5,8,10]. TAMs, which are recruited by inflammatory cytokines and chemokines at the tumor site, are the main players that cause tumor growth and inflammation through the promotion of angiogenesis, cancer cell invasion, and lymph node metastasis in the tumor microenvironment [32]. TAMs are derived from peripheral blood monocytes, and infiltration by TAMs has been linked to growth in various human tumors. They are thought to exert 
these effects by producing growth factors (VEGF) and proinflammatory molecules, such as IL-1 $\beta$, IL-6, TNF- $\alpha$, MMPs, and COX-2 [33]. Various agents, such as PMA and toxins like LPS, have previously been used to induce the differentiation, maturation, and activation of HL-60 and Raw 264.7 cells. Studies have shown that both PMA and LPS bind to the macrophage-specific cell surface receptors CD14 and CD11b, which ultimately results in the phosphorylation and activation of the MAPK/NFkB pathway and the production of inflammatory cytokines [31,34]. Various anti-inflammatory drugs, including the NSAIDs, have been found promising for treating the cancer. These drugs can alter the tumors themselves or the tumor microenvironment, potentially increasing cell death, decreasing migration, and increasing sensitivity of tumors to other therapies [35-37]. For instance, tolfenamic acid has been shown to exert potent anti-tumorigenic activities by regulating multiple molecular targets both in vitro and in nude mouse model [38-42]. DA has been reported to possess anti-inflammatory and anti-tumor activities, such as causing cell cycle arrest, suppressing angiogenesis, and inducing apoptotic cell death in various human tumors [22-24,26]. DA exerts its anti-inflammatory effects by modulating the activation of MMP-9, PI3K, ERK, NFאB, and VEGF in cancer cells [26]. Previously, it was reported that decursin inhibited MMP-9, nitric oxide production and cytokine (IL-8, MCP-1, IL-1 $\beta$, and TNF- $\alpha$ ), which was stimulated by pre-treatment with LPS, TLR-ligands, IL-1 $\beta$, and TNF- $\alpha$ in RAW264.7 cells and THP-1 cells. The study suggested that decursin action was mediated through the inhibition of LPS-induced IкB phosphorylation and NF- $\kappa$ B translocation [43]. Therefore, we investigated the mechanism underlying the activity of DA against macrophage polarization in HL-60 and Raw 264.7 cells. DA significantly blocked macrophage polarization by inhibiting the production of proinflammatory cytokines and activating the MAPK and NFKB pathways. DA disrupted the interplay between cytokines and the MAPK/NFKB pathways in PMA- and LPS-activated macrophages.

Macrophages bidirectionally mediate events between inflammation and cancer development by displaying M1 and M2 phenotypic characteristics. Several reports have highlighted the differential role of M1 and M2 macrophages in various diseases. Macrophages of the M2 phenotype have been reported to promote tumor progression, whereas M1 macrophages have been shown to trigger insulin resistance and atherosclerosis [44]. The excessive production of proinflammatory cytokines such as IL1 and IL6, growth factors, chemokines, and proteases by macrophages results in precancerous lesions, tumor growth promotion, and metastasis. It has been reported that TAMs exhibit characteristics of M2 macrophages in the early stages of tumorigenesis and exert immunosuppressive effects [45,46]. Human HL-60 cells are widely used as an excellent model of the differentiation of monocytes to macrophages. When we treated HL-60 cells with PMA, they attached to the plate and differentiated into macrophages, which was accompanied by an increase in the expression of IL-1 and IL-6 (Figure 1B,C). Treatment with PMA also activated MAPK and NFKB in HL-60 and RAW 264.7 cells (Figure 2A,C). The activation of MAPKs and NFKB induces the transcription of pro-inflammatory cytokines, including IL1 $\beta$, IL-6, and IL-12 [47,48]. The induction of IL-6 by IL1 $\beta$ is mediated by MAPKs and NFkB during inflammation. Our results are consistent with previous reports in showing that IL-1 $\beta$ increased the secretion of IL-6 in response to PMA (Figure 1C). DA effectively blocked the PMA-induced activation of the NFKB and MAPK pathways as well as the production of pro-inflammatory cytokines, such as IL-1 $\beta$ and IL-6, in HL-60 and RAW 264.7 cells.

The bacterial endotoxin LPS is a known agonist of TLR2 that activates the expression of proinflammatory cytokines and the phosphorylation of MAPKs and NFkB [49]. In addition, the activation of the MAPK and NFKB signaling cascades drive inflammation and macrophage polarization. The results of the present study show that the activation of MAPK and NFkB is involved in the modulation of NOX and iNOS expression in LPS-stimulated RAW 264.7 macrophages (Figure 3C). DA treatment not only blocked the differentiation of macrophages by inhibiting NOX-mediated iNOS generation, but also inhibited the activation of IL-1 $\beta$ and IL-6 in LPS-stimulated RAW 264.7 cells (Figure 3B). During macrophage differentiation, when there is an increase in the abundance of the cell surface marker CD11b upon translocation to the plasma membrane, NOX primarily regulates the production of ROS [50,51]. In LPS-induced signal transduction, ROS may serve as a secondary 
messenger, facilitating the regulation of downstream pathways such as NFKB and MAPK, which eventually promote the expression of proinflammatory genes [52]. DA treatment reduced the expression of the M1-specific marker iNOS and the M2-specific marker CD11b, thereby blocking the polarization of macrophages (Figure 3C). Given the differential role of MAPKs in macrophage polarization, we also highlight the fact that LPS-induced differentiation was mainly dependent on the ERK pathway, with negligible input from the p38 or JNK pathway (Figure 2D). On the other hand, NFKB translocation was blocked by DA treatment, suggesting that the potential anti-inflammatory effects of DA are mediated by its ability to regulate macrophage phenotype characteristics (Figure 4B).

Inflammation has been shown to be closely associated with an increase in the incidence of various diseases, including cancer. Interleukins (IL-1 $\beta /$ IL-6), which are key players in inflammation, mediate macrophage polarization by promoting the activity of the NFKB and MAPK signaling pathways and the overproduction of NOX and iNOS. Our results show that DA, which is isolated from the roots of $A$. gigas, has the potential to inhibit inflammatory responses during macrophage differentiation. This anti-inflammatory effect on macrophages is expected to contribute to the anti-cancer activity of DA. We found that treatment with DA suppressed macrophage polarization by inhibiting the production of inflammatory cytokines and free radicals and by activating the NFKB and MAPK pathways. These results suggest that DA might be of therapeutic importance in other inflammatory diseases, but its detailed mechanism of action needs to be investigated.

\section{Materials and Methods}

\subsection{Chemicals and Reagents}

DA with molecular weight 328 was isolated from the roots of Angelicae gigas at Daegu Hanny University, Daegu, Korea as described previously [22]. LPS (cat\# L 2630) and PMA (cat\# P1585) were purchased from Sigma-Aldrich (St. Louis, MO, USA). Mounting medium with DAPI (cat\# H-1200) was obtained from Vector Laboratories, Inc. (Burlingame, CA, USA). Electrophoresis reagents and the Bio-Rad protein assay kit were purchased from Bio-Rad (Hercules, CA, USA). Antibodies were obtained from Santa Cruz Biotechnology (Dallas, TX, USA), Cell Signaling Technology (Danvers, MA, USA), Abcam (Cambridge, UK), and Life Technologies Corporation (Carlsbad, CA, USA). ECL Western blotting detection reagents and nitrocellulose membrane were obtained from Amersham Pharmacia Biotech (Little Chalfont, Buckinghanshire, UK). Superscript III First-Strand Synthesis System kit was purchased from Invitrogen Life Technologies (Waltham, MA, USA). Antibodies were obtained from Santa Cruz Biotech (p65 (sc-372), p50 (sc-1190), IkB $\alpha$ (sc-1643), p-Raf (sc-271929), ERK (sc-292838), p-ERK (sc-7383), JNK (sc-7345), p-JNK (sc-6254), p-38 (sc-271120), and p-p38 (sc-101759)) and Cell Signaling Technology (MEK (cat\# 9122), p-MEK (cat\# 9121), GAPDH (cat\# 2118), and PCNA (cat\# 13110)). All chemicals were stored and used according to the manufacturer's instructions.

\subsection{Cell. Culture and Treatment}

Human leukemia HL-60 (ATCC \#CCL-240) and mouse macrophage RAW 264.7 cells (ATCC \#TIB-71) were cultured in Iscove's Modified Dulbecco's Medium (IMDM, Gibco \#12440-053) and Dulbecco's Modified Eagle Medium (DMEM, Gibco \#11995-065), respectively. Both media were supplemented with 10\% Fetal Bovine Serum (Gibco \#16000-044) and 1\% penicillin-streptomycin (Gibco \#15140-122). Cell cultures were maintained in a humidified incubator containing $5 \% \mathrm{CO}_{2}$ at $37^{\circ} \mathrm{C}$. HL-60 cells were treated with $100 \mathrm{nM}$ PMA for $48 \mathrm{~h}$ to induce cellular differentiation into macrophages $\left(\mathrm{M}_{0}\right)$. After differentiation, cells were treated with varying concentrations of DA $(0,20$, 30 , and $40 \mu \mathrm{M})$. The cells were then collected and washed with PBS to remove cell debris and particles. Similarly, Raw 264.7 macrophages were incubated for $24 \mathrm{~h}$ with LPS $(1 \mu \mathrm{g} / \mathrm{mL})$ with or without exposure to DA. 


\subsection{Nuclear and Cytoplasmic Fractionation}

Cells were cultured at a density of $2 \times 10^{5}$ cells $/ \mathrm{mL}$, and once $70 \%$ confluency was reached, cells were pretreated with LPS and incubated with DA for $24 \mathrm{~h}$. Cells were harvested using trypsin-EDTA, collected through centrifugation, and washed thrice with cold PBS. Fractions were prepared using a Nuclear Extraction Kit (Cayman Chemicals, Ann Arbor, MI, USA) according to the manufacturer's protocol. Nuclear and cytosolic fractions were stored at $-75^{\circ} \mathrm{C}$.

\subsection{Reverse-Transcription Polymerase Chain Reaction (RT-PCR)}

Total RNA was isolated from the cells using TRIZOL reagent (Invitrogen, Carlsbad, CA, USA) according to the manufacturer's protocol. The Superscript III First-Strand Synthesis System kit was used to isolate and purify mRNA from total RNA according to the manufacturer's instructions. Reverse transcription and subsequent cDNA amplification were performed with an Access RT-PCR Introductory System (Promega, Madison, WI, USA) in an Eppendorf Mastercycler. PCR cycle parameters were as follows: initial denaturation at $98{ }^{\circ} \mathrm{C}$ for $3 \mathrm{~min}$, followed by $30-40$ cycles of denaturation at $95{ }^{\circ} \mathrm{C}$ for $30 \mathrm{~s}$, annealing at $55^{\circ} \mathrm{C}$ for $30 \mathrm{~s}$, and extension at $72{ }^{\circ} \mathrm{C}$ for $30 \mathrm{~s}$, and a final extension step at $72{ }^{\circ} \mathrm{C}$ for $5 \mathrm{~min}$. The sequences of the primers used were as follows: IL-1 $\beta$ forward, 5'-ACAGATGAAGTGCTCCTTCCA-3' , and IL-1 $\beta$ reverse, $5^{\prime}$-GTCGGAGATTCGTAGCTGGAT-3'; IL-4 forward, 5'-CGAGTTGACCGTAACAGACAT-3', and IL-4 reverse, 5' -CGTCTTTAGCCTTTCCAA GAAG-3'; IL-6 forward, 5'-GGTACATCCTCGACGGCATCT-3', and IL-6 reverse, 5'-GTGCCT CTTTGCTGCTTTCAC-3'; IL-10 forward, 5'-GCCTAACATGCTTCGAGATC-3' , and IL-10 reverse, 5'-CTCATGGCTTTGTAGATGCC-3' ; and GADPH forward, 5' -AGGGCTGCTTTTAACTCTGGT-3', and GADPH reverse, 5'-CCCCACTTGATTTTGGAGGGA-3'. The PCR products were analyzed through electrophoresis on a $1 \%$ agarose gel with an EcoDye ${ }^{\mathrm{TM}}$ Nucleic Acid Staining Solution (Biofact Co., Ltd., Daejeon, South Korea). Images were then captured using Wise Capture I-1000 software (Daihan Scientific, Seoul, South Korea).

\subsection{Immunofluorescence Assay}

RAW 264.7 cells were seeded onto a 4-well chamber slide (Lab-Tek\#C7182) at a density of $3 \times 10^{3}$ cells $/ \mathrm{mL}$. The cells were stimulated with LPS and then incubated with different concentrations of DA for the indicated duration. These were then washed with PBS and fixed in 3.7\% formaldehyde for $10 \mathrm{~min}$ at room temperature. Afterwards, the cells were permeabilized with $0.1 \%$ Triton X-100 for $4 \mathrm{~min}$ and washed twice with PBS. Then, the cells were incubated with anti-CD11b, anti-iNOS, or anti-NFKB p65 antibodies (1:100 with $2 \%$ BSA) overnight at $4{ }^{\circ} \mathrm{C}$, followed by incubation with goat anti-rabbit/anti-mouse IgG conjugated to either FITC or Alexa Flour 594 for $1 \mathrm{~h}$ at room temperature in the dark. Finally, the cells were washed with PBS and incubated with DAPI mounting medium for 5-10 min. The samples were then analyzed through fluorescence microscopy.

\subsection{Enzyme-Linked Immunosorbent Assay (ELISA)}

The levels of IL-1 $\beta$ and IL-6 exogenously secreted by the HL-60 and Raw 264.7 cells were determined through ELISA using a kit from Shire (Lexington, MA, USA). Ninety-six-well plates (MaxisorpImmuno-plate; Sigma-Aldrich, St. Louis, MO, USA) were coated with $10 \mu \mathrm{g} / \mathrm{mL}$ purified goat anti-IL-1 $\beta$ / anti-IL-6 antibodies. Cell culture supernatants from the control and treated groups were incubated in these plates for $1 \mathrm{~h}$, and then a polyclonal rabbit anti-IL-1 $\beta /$ IL-6 antibody was added to the wells. HRP-conjugated goat anti-rabbit IgG was then added, and the cells were incubated for 30 min. 2,2'-Azino-bis(3-ethylbenzthiazoline-6-sulfonic acid) (ABTS, Sigma \#A1888) was then added to the plate, and the absorbance at $405 \mathrm{~nm}$ was detected using a reference wavelength of $490 \mathrm{~nm}$. 


\subsection{Western Blot}

After cell lysis, the total protein content was isolated and quantified using the Bradford protein assay. The protein samples were denatured at $95{ }^{\circ} \mathrm{C}$ for $5 \mathrm{~min}$. Next, the samples were analyzed through sodium dodecyl sulfate-polyacrylamide gel electrophoresis (SDS-PAGE) and Western blot using polyvinylidene fluoride microporous membranes. The membranes were incubated with primary antibodies (1:1000 with TBST) overnight at $4{ }^{\circ} \mathrm{C}$, and then were incubated with HRP-conjugated secondary antibodies for $1 \mathrm{~h}$. Protein bands were then detected using an ECL Western blotting detection reagent.

\subsection{Statistical Analysis}

All experiments were performed with triplicate samples, and all experiments were performed at least three times. Data are presented as the mean \pm SD. Comparisons between two groups were analyzed using the Student's $t$-test, while comparisons between more than two groups were analyzed using analysis of variance. Differences with $p$ values $\leq 0.05$ were considered statistically significant.

Author Contributions: Y.S.L. conceived of the presented idea and supervised the work; S.U.I. and J.H.L. collected materials to carry out and performed the experiment; A.S. wrote the manuscript and analyzed the data; E.M.A. and Y.M.L. provided the decursin angelate and other chemicals and reagents as well as contributed to the interpretation of the results.

Funding: This research was funded by the Basic Science Research Program of the NRF funded by the Ministry of Science, ICT, and Future Planning, grant number [NRF-2016R1A2B4012677]. The APC was also funded by it.

Conflicts of Interest: The authors declare no conflict of interest.

\section{References}

1. Pullamsetti, S.; Savai, R.; Janssen, W.; Dahal, B.; Seeger, W.; Grimminger, F.; Ghofrani, H.; Weissmann, N.; Schermuly, R. Inflammation, immunological reaction and role of infection in pulmonary hypertension. Clin. Microbiol. Infect. 2011, 17, 7-14. [CrossRef] [PubMed]

2. Kuper, H.; Adami, H.O.; Trichopoulos, D. Infections as a major preventable cause of human cancer. J. Intern. Med. 2000, 248, 171-183. [CrossRef] [PubMed]

3. Blaser, M.J.; Chyou, P.; Nomura, A. Age at establishment of Helicobacter pylori infection and gastric carcinoma, gastric ulcer, and duodenal ulcer risk. Cancer Res. 1995, 55, 562-565. [PubMed]

4. Scholl, S.; Pallud, C.; Beuvon, F.; Hacene, K.; Stanley, E.; Rohrschneider, L.; Tang, R.; Pouillart, P.; Lidereau, R. Anti-colony-stimulating factor-1 antibody staining in primary breast adenocarcinomas correlates with marked inflammatory cell infiltrates and prognosis. J. Natl. Cancer Inst. 1994, 86, 120-126. [CrossRef] [PubMed]

5. Shacter, E.; Weitzman, S.A. Chronic inflammation and cancer. Oncology (Williston Park, N.Y.) 2002, 16, $217-226$.

6. Maeda, H.; Akaike, T. Nitric oxide and oxygen radicals in infection, inflammation, and cancer. Biochem. Mosc. 1998, 63, 854-865.

7. Yamanishi, Y.; Boyle, D.L.; Rosengren, S.; Green, D.R.; Zvaifler, N.J.; Firestein, G.S. Regional analysis of p53 mutations in rheumatoid arthritis synovium. Proc. Natl. Acad. Sci. USA 2002, 99, 10025-10030. [CrossRef] [PubMed]

8. Gregory, A.D.; Houghton, A.M. Tumor-associated neutrophils: New targets for cancer therapy. Cancer Res. 2011, 71, 2411-2416. [CrossRef] [PubMed]

9. Imtiyaz, H.Z.; Williams, E.P.; Hickey, M.M.; Patel, S.A.; Durham, A.C.; Yuan, L.-J.; Hammond, R.; Gimotty, P.A.; Keith, B.; Simon, M.C. Hypoxia-inducible factor $2 \alpha$ regulates macrophage function in mouse models of acute and tumor inflammation. J. Clin. Investig. 2010, 120, 2699-2714. [CrossRef] [PubMed]

10. Almatroodi, S.A.; McDonald, C.F.; Darby, I.A.; Pouniotis, D.S. Characterization of M1/M2 tumour-associated macrophages (TAMs) and Th1/Th2 cytokine profiles in patients with NSCLC. Cancer Microenviron. 2016, 9, 1-11. [CrossRef] [PubMed] 
11. Van Ginderachter, J.A.; Movahedi, K.; Ghassabeh, G.H.; Meerschaut, S.; Beschin, A.; Raes, G.; De Baetselier, P. Classical and alternative activation of mononuclear phagocytes: Picking the best of both worlds for tumor promotion. Immunobiology 2006, 211, 487-501. [CrossRef] [PubMed]

12. Medzhitov, R. Origin and physiological roles of inflammation. Nature 2008, 454, 428-435. [CrossRef] [PubMed]

13. Martinez, F.O.; Gordon, S. The M1 and M2 paradigm of macrophage activation: Time for reassessment. F1000Prime Rep. 2014, 6, 13. [CrossRef] [PubMed]

14. Rath, M.; Müller, I.; Kropf, P.; Closs, E.I.; Munder, M. Metabolism via arginase or nitric oxide synthase: Two competing arginine pathways in macrophages. Front. Immunol. 2014, 5, 532. [CrossRef] [PubMed]

15. Chanmee, T.; Ontong, P.; Konno, K.; Itano, N. Tumor-associated macrophages as major players in the tumor microenvironment. Cancers 2014, 6, 1670-1690. [CrossRef] [PubMed]

16. Siegel, R.L.; Miller, K.D.; Jemal, A. Cancer statistics, 2017. CA Cancer J. Clin. 2017, 67, 7-30. [CrossRef] [PubMed]

17. Rakoff-Nahoum, S.; Medzhitov, R. Toll-like receptors and cancer. Nat. Rev. Cancer 2009, 9, 57-63. [CrossRef] [PubMed]

18. Rafa, H.; Benkhelifa, S.; AitYounes, S.; Saoula, H.; Belhadef, S.; Belkhelfa, M.; Boukercha, A.; Toumi, R.; Soufli, I.; Moralès, O. All-trans retinoic acid modulates TLR4/NF-kB signaling pathway targeting TNF- $\alpha$ and nitric oxide synthase 2 expression in colonic mucosa during ulcerative colitis and colitis associated cancer. Mediat. Inflamm. 2017, 2017, 7353252. [CrossRef] [PubMed]

19. Nyati, K.K.; Masuda, K.; Zaman, M.M.-U.; Dubey, P.K.; Millrine, D.; Chalise, J.P.; Higa, M.; Li, S.; Standley, D.M.; Saito, K. TLR4-induced NF-кB and MAPK signaling regulate the IL-6 mRNA stabilizing protein Arid5a. Nucleic Acids Res. 2017, 45, 2687-2703. [CrossRef] [PubMed]

20. Chan, E.D.; Riches, D.W. IFN- $\gamma+$ LPS induction of iNOS is modulated by ERK, JNK/SAPK, and p38 mapk in a mouse macrophage cell line. Am. J. Physiol. Cell. Physiol. 2001, 280, C441-C450. [CrossRef] [PubMed]

21. Soufli, I.; Toumi, R.; Rafa, H.; Touil-Boukoffa, C. Overview of cytokines and nitric oxide involvement in immuno-pathogenesis of inflammatory bowel diseases. World J. Gastrointest. Pharmacol. Ther. 2016, 7, 353-360. [CrossRef] [PubMed]

22. Jung, M.H.; Lee, S.H.; Ahn, E.-M.; Lee, Y.M. Decursin and decursinol angelate inhibit VEGF-induced angiogenesis via suppression of the VEGFR-2-signaling pathway. Carcinogenesis 2009, 30, 655-661. [CrossRef] [PubMed]

23. Lee, S.; Lee, Y.S.; Jung, S.H.; Shin, K.H.; Kim, B.-K.; Kang, S.S. Anti-tumor activities of decursinol angelate and decursin from Angelica gigas. Arch. Pharm. Res. 2003, 26, 727-730. [CrossRef] [PubMed]

24. Kim, H.H.; Bang, S.S.; Choi, J.S.; Han, H.; Kim, I.-H. Involvement of PKC and ROS in the cytotoxic mechanism of anti-leukemic decursin and its derivatives and their structure-activity relationship in human K562 erythroleukemia and U937 myeloleukemia cells. Cancer Lett. 2005, 223, 191-201. [CrossRef] [PubMed]

25. Shehzad, A.; Parveen, S.; Qureshi, M.; Subhan, F.; Lee, Y.S. Decursin and decursinol angelate: Molecular mechanism and therapeutic potential in inflammatory diseases. Inflamm. Res. 2018, 67, 209-218. [CrossRef] [PubMed]

26. Kim, W.-J.; Lee, M.-Y.; Kim, J.-H.; Suk, K.; Lee, W.-H. Decursinol angelate blocks transmigration and inflammatory activation of cancer cells through inhibition of PI3K, ERK and NF-kB activation. Cancer Lett. 2010, 296, 35-42. [CrossRef] [PubMed]

27. Son, S.H.; Kim, M.-J.; Chung, W.-Y.; Son, J.-A.; Kim, Y.S.; Kim, Y.-C.; Kang, S.S.; Lee, S.-K.; Park, K.-K. Decursin and decursinol inhibit VEGF-induced angiogenesis by blocking the activation of extracellular signal-regulated kinase and c-Jun N-terminal kinase. Cancer Lett. 2009, 280, 86-92. [CrossRef] [PubMed]

28. Mosser, D.M. The many faces of macrophage activation. J. Leukoc. Biol. 2003, 73, 209-212. [CrossRef] [PubMed]

29. Sica, A.; Mantovani, A. Macrophage plasticity and polarization: In vivo veritas. J. Clin. Investig. 2012, 122, 787-795. [CrossRef] [PubMed]

30. Cahill, C.M.; Rogers, J.T. Interleukin (IL) $1 \beta$ induction of IL-6 is mediated by a novel phosphatidylinositol 3-kinase-dependent AKT/IкB kinase $\alpha$ pathway targeting activator protein-1. J. Biol. Chem. 2008, 283, 25900-25912. [CrossRef] [PubMed] 
31. Zheng, X.-F.; Hong, Y.-X.; Feng, G.-J.; Zhang, G.-F.; Rogers, H.; Lewis, M.A.; Williams, D.W.; Xia, Z.-F.; Song, B.; Wei, X.-Q. Lipopolysaccharide-induced M2 to M1 macrophage transformation for IL-12p70 production is blocked by Candida albicans mediated up-regulation of EBI3 expression. PLOS ONE 2013, 8, e63967. [CrossRef] [PubMed]

32. Yang, J.; Li, X.; Liu, X.; Liu, Y. The role of tumor-associated macrophages in breast carcinoma invasion and metastasis. Int. J. Clin. Exp. Pathol. 2015, 8, 6656-6664. [PubMed]

33. Smith, H.A.; Kang, Y. The metastasis-promoting roles of tumor-associated immune cells. Int. J. Mol. Med. 2013, 91, 411-429. [CrossRef] [PubMed]

34. Song, M.-g.; Ryoo, I.-G.; Choi, H.-y.; Choi, B.-h.; Kim, S.-T.; Heo, T.-H.; Lee, J.Y.; Park, P.-H.; Kwak, M.-K. NRF2 signaling negatively regulates phorbol-12-myristate-13-acetate (PMA)-induced differentiation of human monocytic U937 cells into pro-inflammatory macrophages. PLoS ONE 2015, 10, e0134235. [CrossRef] [PubMed]

35. Zlotnik, A. Involvement of Chemokine Receptors in Organ-Specific Metastasis. In Infection and Inflammation: Impacts on Oncogenesis; Karger Publishers: Basel, Switzerland, 2006; Volume 13, pp. 191-199.

36. Jana, N.R. NSAIDs and apoptosis. Cell. Mol. Life Sci. 2008, 65, 1295-1301. [CrossRef] [PubMed]

37. De Groot, D.; De Vries, E.; Groen, H.; De Jong, S. Non-steroidal anti-inflammatory drugs to potentiate chemotherapy effects: From lab to clinic. Crit. Rev. Oncol. Hematol. 2007, 61, 52-69. [CrossRef] [PubMed]

38. Zhang, X.; Lee, S.-H.; Min, K.-W.; McEntee, M.F.; Jeong, J.; Li, Q.; Baek, S.J. The involvement of endoplasmic reticulum stress in the suppression of colorectal tumorigenesis by tolfenamic acid. Cancer Prev. Res. (Phila.) 2013, 6, 1337-1347. [CrossRef] [PubMed]

39. Abdelrahim, M.; Baker, C.H.; Abbruzzese, J.L.; Safe, S. Tolfenamic acid and pancreatic cancer growth, angiogenesis, and Sp protein degradation. J. Natl. Cancer Inst. 2006, 98, 855-868. [CrossRef] [PubMed]

40. Abdelrahim, M.; Baker, C.H.; Abbruzzese, J.L.; Sheikh-Hamad, D.; Liu, S.; Cho, S.D.; Yoon, K.; Safe, S. Regulation of vascular endothelial growth factor receptor- 1 expression by specificity proteins 1,3 , and 4 in pancreatic cancer cells. Cancer Res. 2007, 67, 3286-3294. [CrossRef] [PubMed]

41. Chadalapaka, G.; Jutooru, I.; Sreevalsan, S.; Pathi, S.; Kim, K.; Chen, C.; Crose, L.; Linardic, C.; Safe, S. Inhibition of rhabdomyosarcoma cell and tumor growth by targeting specificity protein (Sp) transcription factors. Int. J. Cancer 2013, 132, 795-806. [CrossRef] [PubMed]

42. Colon, J.; Basha, M.R.; Madero-Visbal, R.; Konduri, S.; Baker, C.H.; Herrera, L.J.; Safe, S.; Sheikh-Hamad, D.; Abudayyeh, A.; Alvarado, B. Tolfenamic acid decreases c-Met expression through Sp proteins degradation and inhibits lung cancer cells growth and tumor formation in orthotopic mice. Investig. New Drugs 2011, 29, 41-51. [CrossRef] [PubMed]

43. Kim, J.-H.; Jung, J.-H.; Jeon, S.-T.; Kim, H.; Ock, J.; Suk, K.; Kim, S.-I.; Song, K.-S.; Lee, W.-H. Decursin inhibits induction of inflammatory mediators by blocking NF-kB activation in macrophages. Mol. Pharmacol. 2006, 69, 1783-1790. [CrossRef] [PubMed]

44. Tan, H.-Y.; Wang, N.; Li, S.; Hong, M.; Wang, X.; Feng, Y. The reactive oxygen species in macrophage polarization: Reflecting its dual role in progression and treatment of human diseases. Oxid. Med. Cell. Longev. 2016, 2016, 2795090. [CrossRef] [PubMed]

45. Williams, C.B.; Yeh, E.S.; Soloff, A.C. Tumor-associated macrophages: Unwitting accomplices in breast cancer malignancy. NPJ Breast Cancer 2016, 2, 15025. [CrossRef] [PubMed]

46. Quatromoni, J.G.; Eruslanov, E. Tumor-associated macrophages: Function, phenotype, and link to prognosis in human lung cancer. Am. J. Transl. Res. 2012, 4, 376-389.

47. Hayden, M.S.; Ghosh, S. Signaling to NF-kappaB. Genes Dev. 2004, 18, 2195-2224. [CrossRef] [PubMed]

48. Yang, Y.; Cui, J.; Gao, F.; Li, B.; Liu, C.; Zhang, P.; Huang, Y.; Liu, W.; Liu, H.; Cai, J. Whole Body Irradiation Induces Cutaneous Dendritic Cells Depletion via NF-кB Activation. Cell. Physiol. Biochem. 2013, 32, $200-209$. [CrossRef] [PubMed]

49. Guha, M.; Mackman, N. LPS induction of gene expression in human monocytes. Cell Signal. 2001, 13, 85-94. [CrossRef]

50. Kim, J.B.; Han, A.R.; Park, E.Y.; Kim, J.Y.; Cho, W.; Lee, J.; Seo, E.K.; Lee, K.T. Inhibition of LPS-induced iNOS, COX-2 and cytokines expression by poncirin through the NF-kappaB inactivation in RAW 264.7 macrophage cells. Biol. Pharm. Bull. 2007, 30, 2345-2351. [CrossRef] [PubMed] 
51. Zhao, K.; Huang, Z.; Lu, H.; Zhou, J.; Wei, T. Induction of inducible nitric oxide synthase increases the production of reactive oxygen species in RAW264. 7 macrophages. Biosci. Rep. 2010, 30, 233-241. [CrossRef] [PubMed]

52. Kohchi, C.; Inagawa, H.; Nishizawa, T.; Soma, G.-I. ROS and innate immunity. Anticancer Res. 2009, 29, 817-821. [PubMed]

Sample Availability: Samples of the compounds are all available from the authors.

(C) 2018 by the authors. Licensee MDPI, Basel, Switzerland. This article is an open access article distributed under the terms and conditions of the Creative Commons Attribution (CC BY) license (http://creativecommons.org/licenses/by/4.0/). 